Relations industrielles

Industrial Relations

\title{
Les horaires flexibles
}

\section{Flexible Working Hours}

\section{Pierrette Sartin}

Volume 29, numéro 2, 1974

URI : https://id.erudit.org/iderudit/028508ar

DOI : https://doi.org/10.7202/028508ar

Aller au sommaire du numéro

Éditeur(s)

Département des relations industrielles de l'Université Laval

ISSN

0034-379X (imprimé)

1703-8138 (numérique)

Découvrir la revue

Citer cet article

Sartin, P. (1974). Les horaires flexibles. Relations industrielles / Industrial Relations, 29(2), 343-365. https://doi.org/10.7202/028508ar
Résumé de l'article

L'auteur présente les horaires flexibles, leurs avantages pour l'employeur et pour l'employé, leurs modalités d'application et les réactions de différentes parties à cette forme d'aménagement du temps de travail.
Tous droits réservés (C) Département des relations industrielles de l'Universite Laval, 1974
Ce document est protégé par la loi sur le droit d'auteur. L'utilisation des services d'Érudit (y compris la reproduction) est assujettie à sa politique d'utilisation que vous pouvez consulter en ligne.

https://apropos.erudit.org/fr/usagers/politique-dutilisation/ 


\section{Les horaires flexibles}

\section{Pierrette Sartin}

L'auteur présente les horaires flexibles, leurs avantages pour l'employeur et pour l'employé, leurs modalités d'application et les réactions de différentes parties à cette forme d'aménagement du temps de travail.

Jusqu'à ces dernières années les entreprises ont vécu sur le mode des horaires imposés et rigides. Ces horaires étaient fixés par la loi ou par des conventions et le salarié était tenu de les respecter strictement, qu'il $\mathrm{y}$ ait ou non obligation de pointage.

Mais depuis cinq années environ, les entreprises se sont efforcées de desserrer les contraintes souvent inutiles que représentaient ces horaires rigides et d'adapter les heures de travail aux vœux de chacun grâce à un horaire plus souple.

Les premières expériences furent faites en Allemagne et en Suisse. Elles ont gagné d'autres pays et en Europe aujourd'hui, la Grande Bretagne, la Suède, le Luxembourg, les Pays-Bas et la France tendent à appliquer ce genre d'horaires. En Allemagne fédérale, plus de 2 millions de salariés les pratiquent déjà et l'on pense que prochainement $80 \%$ de la population salariale sera touchée par ceux-ci. En France plusieurs entreprises ont tenté l'expérience : l'entreprise Helmer à Colmar, les produits pharmaceutiques Roche, des services administratifs de Peugeot, l'Union des caisses centrales de la mutualité agricole, les assurances, les banques etc....

On les trouve maintenant appliqués dans l'industrie des métaux, le textile, les industries alimentaires, chimiques, le commerce, les assurances et certaines administrations publiques.

Le principe de base est le choix libre et individuel des heures d'arrivée et de départ à l'intérieur de certaines limites.

SARTIN, Pierrette, Commissariat au Plan d'équipement et de productivité, Paris. 
Ces horaires doivent respecter deux impératifs :

- un temps de présence obligatoire quotidien et fixe pour tous les travailleurs

- une durée de travail conforme à la durée hebdomadaire légale. DURÉE QUOTIDIENNE DU TRAVAIL

Ce système consiste généralement à distinguer dans la durée quotidienne du travail, 3 parties :

Une partie fixe, dite PLAGE FIXE, au cours de laquelle l'ensemble $\mathrm{du}$ personnel doit être présent.

Deux parties variables, dites PLAGES MOBILES, au début et à la fin de la journée. Parfois au milieu de la journée lors de la pause $\mathrm{du}$ repas, on instaure une autre plage. Mais celle-ci ne doit pas être entièrement libre et dans l'intérêt même des travailleurs, un temps d'arrêt de 30 à 45 minutes au moins doit leur être imposée pour le repas.

La durée de chaque partie variable doit être d'au moins 1 heure (dans certaines entreprises elle n'est que de 10 minutes ce qui est nettement insuffisant).

Mais il est préférable de laisser $1 \mathrm{~h} \quad 1 / 2$ ou 2 heures de latitude au personnel pour le choix de son horaire.

- Dans certaines entreprises, il n'existe que des PLAGES mobiles. Les salariés arrivent et partent quand ils veulent, à condition que l'horaire hebdomadaire soit respecté. Mais un tel système ne se conçoit que dans des entreprises particulières (laboratoires de recherches par exemple travaux de conception etc. ... où la liberté totale ne risque pas d'être une entrave au travail des autres membres du personnel.

\section{AVANTAGES}

Les horaires libres sont jusqu'à présent la seule amélioration apportée aux conditions de travail qui soit à la fois bénéfique pour l'employeur et pour les salariés.

Pour l'employeur elle se traduit :

a) par la facilité de comptabiliser les heures fournies par le personnel grâce à la simple lecture optique des indications enregistrées sur les appareils de contrôle individuels.

b) par une baisse de l'absentéisme de courte durée. 
c) par une réduction des embouteillages internes (rencontre dans les couloirs, attente aux ascenseurs etc.).

d) par une diminution des accidents du trajet.

e) par une baisse des heures supplémentaires, grâce à un meilleur équilibrage du temps de présence.

Chez VOKO, usine de meubles de Bureau à Giesen (Allemagne) les collaborateurs s'efforcent de limiter les heures supplémentaires : le temps excédentaire dépassant 6 heures par mois est annulé. En revanche, les déficits de temps dépassant sont reportés intégralement au mois suivant et doivent être compensés par la perte d'une journée de congé annuel. De plus, le collaborateur est prié par son supérieur de compenser son déficit dans les meilleurs délais.

f) par une diminution du turn over (de 2 à $5 \%$ en Suisse sur un taux moyen de $20 \%$ ).

g) par une facilité plus grande d'embauche. On a constaté dans l'industrie privée que les demandeurs d'emploi se présentaient de préférence dans les entreprises pratiquant les horaires libres et ceux-ci sont parfois mentionnés dans les petites annonces.

h) dans de nombreux cas on trouve avantage à ce que, grâce aux plages mobiles, l'entreprise ou les services soient ouverts plus longtemps. On a en effet observé dans de nombreuses entreprises qu'une partie importante du personnel préférait arriver plus tôt et partir plus tôt et notamment cherchait à gagner du temps pour dégager la plage mobile du vendredi soir.

i) pour les cadres, la plage mobile constitue un moment où ils peuvent travailler dans le calme.

De plus, certaines personnes préférant venir tôt le matin, d'autres partir tard le soir, les chefs de service n'ont besoin de faire aucune pression pour que leurs collaborateurs soient présents hors des limites de l'ancien horaire.

j) pour les cadres la plage mobile constitue un moment où ils peuvent travailler dans le calme.

k) en ce qui concerne l'amélioration de la productivité, elle est difficile à chiffrer. Elle a pu l'être en Suisse chez Brown Boveri où sur 26 chefs de département 5 ont conclu à une amélioration du rendement, 1 à une baisse et 20 à la stabilité.

En France les expériences sont en général trop récentes. Cependant à Colmar, l'entreprise Helmer qui pratique depuis près de 2 ans ces 
horaires a pu réduire de $3 / 4$ d'heure par jour son temps de travail tout en augmentant de 30 à $35 \%$ son chiffre d'affaires.

1) une amélioration du climat social, le personnel étant plus détendu et n'étant plus culpabilisé par les retards ou humilié par les observations et les sanctions ou par la nécessité de demander une autorisation accordée comme une faveur.

m) une diminution des conflits internes et une amélioration des rapports entre le personnel subalterne et les cadres moyens, ceux-ci n'ayant plus l'occasion de se livrer à des contrôles tatillons ou de sanctionner les retards.

Chacun étant responsable de l'organisation de son temps de présence est mieux disposé à organiser ses tâches. Il n'est pas rare de voir une secrétaire terminer le soir une lettre qu'elle aurait laissée inachevée avant, sous prétexte qu'il était l'heure de partir.

n) un sens plus grand des responsabilités chez le personnel. On a $\mathrm{pu}$ ainsi constater en Suisse que les problèmes d'organisation interne se réglaient d'eux-mêmes. Les banques ayant demandé que soient désignés d'avance des équipes de volontaires pour assurer le service le vendredi soir jusqu'à 19 h 30 personne ne se présenta. Le personnel refusa de donner des noms et de prévoir à l'avance les équipes, et prétendit s'organiser lui-même. La direction accepta et aucune difficulté n'a surgi. D'autres entreprises ont fait des expériences analogues. La prise de conscience des responsabilités qui accompagne l'autogestion du temps tend à supprimer les organisations directives et rejoint le soucci pragmatisme dont nous parlerons plus loin.

Pour les employés

Les horaires variables ont entraîné :

a) un gain de temps souvent très important sur les transports en permettant une meilleure harmonisation entre les horaires des transports collectifs et l'heure d'arrivée ou de départ et en économisant une partie du temps perdu par l'encombrement du trafic. Dans de nombreux cas on note un gain de 30 à 45 minutes.

b) une harmonisation de ces horaires aux besoins familiaux ou personnels (heures d'ouverture des écoles, des crèches, réveils matinaux plus ou moins faciles etc....). Chaque individu a son rythme de vie ; chaque famille aussi selon qu'elle a ou non des enfants en âge scolaire.

c) une diminution de la fatigue nerveuse due à la course à la montre et à l'angoisse du retard. 
d) mais l'amélioration la plus sensible est un changement dans les mentalités auquel doit d'ailleurs correspondre un changement dans la mentalité directoriale. Les rapports entre salariés et employeurs changent de forme. L'employeur ou les directions abandonnent une partie de leurs prérogatives, celle d'imposer, comme ils en ont le droit reconnu par la loi, un horaire fixe ; le salarié, de son côté s'engage non plus à respecter des horaires, mais à accomplir un certain nombre d'heures de travail.

Il ne doit plus ni se considérer ni être considéré comme un salarié, mais comme un prestataire de service, ayant à fournir un temps forfaitaire de travail.

Cela implique évidemment un changement radical dans les mentalités des directions et employeurs, comme dans celle des salariés. Mais il ne faut pas perdre de vue que présence ne signifie nullement travail et qu'il vaut mieux juger les gens sur leur efficacité que sur le nombre d'heures effectuées ; ce qui est beaucoup plus difficile.

Ce qui importe dans l'horaire libre c'est que la tâche soit accomplie dans un certain délai et non le moment précis où elle le sera.

Il ne faut pas non plus se dissimuler que l'horaire variable n'est qu'un premier pas dans une nouvelle voie du management et de la gestion du personnel. C'est un début et non un terme. Les Suisses en sont parfaitement concients et pensent que quand cette expérience aura fait ses preuves, à l'avenir, les salariés demanderont d'autres responsabilités et les méthodes de gestion devront évoluer dans ce sens. Le personnel ira vers un refus de plus en plus grand des contraintes et vers une recherche de plus en plus grande de l'autonomie!

Il semble bien que le processus soit inéluctable et irréversible (sauf bien entendu dans le cas d'une réduction massive de la durée du travail qui rendrait les horaires libres inutiles).

Ajoutons que sur un plan plus général, ces horaires constituent le mode vainement cherché de réduction des embouteillages et des heures de pointe, et que sur le plan de l'hygiène mentale et de la physiologie des travailleurs ils constitueront un grand progrès grâce à la suppression de l'angoisse et de toutes les occasions de 'stress' qui accompagnaient les horaires rigides (course à la montre, énervement, tensions, mauvais sommeil, petit déjeuner pris à la hâte ou inexistant etc....). 


\section{LES MODALITÉS D'APPLICATION}

Le système comporte plusieurs possibilités :

- ou bien le maintien de la durée quotidienne du travail : le salarié ne peut pas reporter d'un jour sur l'autre son avance ou son retard. Avec un horaire quotidien de 9 heures par exemple (pause du midi comprise), si la partie variable va de 8 à 10 heures et de 17 à 19 heures, le salarié qui arrive à $8 \mathrm{~h} 10$ devra partir à $17 \mathrm{~h} 10$.

- ou bien le maintien de la durée hebdomadaire de travail.

- le salarié peut travailler plus longtemps certains jours et gagner un crédit d'heures dont il disposera un autre jour de la semaine ou l'inverse.

La durée de la pause de midi peut être également rendue variable. Mais il faut prendre soin de respecter la législation qui interdit aux jeunes de moins de 18 ans et aux femmes de faire respectivement plus de $8 \mathrm{~h}$ et de 10 heures de travail par jour.

En ce qui concerne le choix de ces horaires plusieurs possibilités sont offertes :

- ou bien le personnel choisit une fois pour toute ses heures d'arrivée et de départ. Ce qui dans une large mesure limite sa liberté.

- ou il indique la semaine précédente les horaires qu'il respectera la semaine suivante (nous verrons que c'est là un moyen d'éviter les ennuis avec la sécurité sociale en cas d'accident de trajet).

- ou il existe 2 ou 3 horaires différents d'arrivée et de départ et le salarié en choisit un auquel il se tient pendant une certaine période.

- ou bien les heures d'arrivée et de départ sont entièrement libres dans la limite de la plage fixe et généralement sous réserve que les salariés se soient mis d'accord entre eux pour qu'il y ait toujours quelqu'un de présent dans le service.

Comme on le voit il existe de nombreuses formules d'horaires flexibles.

On a pu remarquer dans de nombreuses entreprises que dès la mise en place de l'expérience plus de $50 \%$ du personnel avait changé d'horaire. Ce qui tend à montrer que cette réforme était nécessaire.

On remarque aussi que les personnes âgées arrivent en général à l'horaire ancien et n'utilisent les possibilités offertes que 2 ou 3 fois par mois. Mais les jeunes les utilisent beaucoup plus surtout (et contrai- 
rement à ce que l'on pourrait croire) le personnel masculin. La liberté des horaires est moins grande chez les femmes en raison des contraintes de leur vie familiale (heures d'ouverture des crèches, de l'école etc....).

\section{Reports et heures supplémetaires}

Un certain nombre d'entreprises permettent à leur personnel de 'reporter' sur la semaine suivante, voire sur le mois, ou sur l'année le nombre d'heures dont il est créditeur ou redevable.

Toutefois le crédit ou le débit d'heures ne doit pas dépasser 10, parfois 15 heures (mais rarement) par quinzaine ou par mois. Au-delà de cette limite le crédit n'est plus récupérable et le débit peut donner lieu à une retenue de salaire.

Psychologiquement et pratiquement, ce report étalé sur une durée excédant la semaine est plus favorable que le report quotidien ou hebdomadaire car il laisse une liberté plus grande. Mais en même temps il risque de mettre l'employeur en infraction avec la législation du travail : d'une part s'il dépasse le nombre d'heures «supplémentaires » permises, d'autre part si, comme c'est généralement le cas, il ne paye pas comme heures supplémentaires, ces heures qui sont en réalité des heures de récupération. En effet, les heures travaillées par semaine, au delà des $40 \mathrm{~h}$ légales sont considérées comme des heures supplémentaires. Un salarié pratiquant l'horaire flexible peut travailler 30 heures pendant une semaine et 50 heures la semaine suivante. Il n'aura accompli qu'une moyenne de 40 heures en mettant à profit les facilités que lui donne son employeur. Cependant, il est hors de doute qu'il serait en droit légalement de réclamer le paiement de 10 heures supplémentaires, la semaine civile étant la seule unité de temps de travail reconnue par la législation français.

Le respect strict de la législation voudrait donc que l'on restreignit les libertés d'horaires à la semaine et que l'on prescrivit les reports. Même dans le cas ou un accord ou une convention prévoient des dispositions contraires, elles n'ont aucune valeur car elles sont en contradiction avec une loi d'ordre public.

Dans le cas où un salarié demanderait à ce que ces heures effectuées au-delà de $40 \mathrm{~h}$ soient payées au tarif des heures supplémentaires, le Tribunal des Prudhommes admettrait sans nul doute que sa demande est fondée. Cependant il semble que jusqu'ici cette éventualité n'ait pro- 
voqué aucun contentieux. Mais le problème des heures supplémentaires est une des raisons des réticences du ministère du travail et des syndicats devant l'application des horaires flexibles. Ils craignent en effet des abus dans la mesure où un employeur prévoyant un surcroit de travail pour la semaine à venir, peut inciter ses salariés à faire moins que l'horaire obligatoire pendant la semaine qui précède, pour 'récupérer' la semaine suivante au lieu d'avoir recours aux heures supplémentaires payées au tarif légal.

En ce qui concerne les heures réellement effectuées en plus des heures normales la pratique dans les entreprises est de ne les admettre et de ne les rémunérer que si elles ont été expressément demandées par l'employeur et non si elles ont été faites à la seule initiative du salarié.

En ce qui concerne l'administration, le problème se pose sous une forme un peu différente. Mais, au niveau des cadres notamment, des exigences pourront apparaître sous la forme d'heures de récupération.

Dans les entreprises la règle adoptée est de ne pas transformer le cumul d'heures supplémentaires en jour de congé, et il n'est pas admis d'employer son crédit d'heures pour réduire son horaire journalier en deça de l'horaire fixe.

Certaines entreprises envisagent néanmoins de s'ouvrir à cette possibilité. Mais cette tolérance ne serait admise qu'après une pratique plus longue du système actuel.

\section{Le choix des horaires : le contrôle.}

L'horaire libre suppose un contrôle. La maison Suchard à Serrières, (en Suisse) qui avait tenté d'établir cet horaire sans contrôle a dû en instaurer un à la demande du personnel lui-même, excédé de voir les fraudeurs qui ne respectaient pas le temps de travail, bénéficier de l'impunité.

Toutefois ce contrôle doit être aussi souple et discret que possible et ne pas revêtir les aspects d'un contrôle policier. Il s'agit en fait d'apprendre aux salariés à acquérir une mentalité d'adultes et à gérer eux-mêmes le temps qu'ils doivent à l'entreprise.

Chez Bergerat-Monnoyeur, agent de Caterpillar en France, qui emploie 3000 personnes, avant l'application des horaires libres, le contrôle était fait par un huissier. Maintenant il existe un auto-contrôle. Une 
batterie de compteurs individuels miniaturisés a été répartie entre les différents étages de l'immeuble. Chaque personne, en arrivant à son lieu de travail, introduit une clé ou badge, en matière plastique, libellée à son nom, dans une fente qui porte également son nom. Une petite lampe témoin s'allume et un compteur individuel enregistre non pas le temps d'arrivée mais la durée du travail de chacun. Le badge reste inséré tant que la personne à qui il appartient est présente dans l'entreprise. Les compteurs individuels sont remis à zéro une fois par mois et chaque personne peut suivre quotidiennement le cumul de ses heures de présence. De plus, chaque mois, au-dessus du compteur est affichée une grille qui permet de comparer les heures effectuées réellement avec les heures théoriques moyennes.

Toutes les entreprises insistent sur le fait que ce système repose sur la confiance.

C'est pourquoi le contrôle est fait en général par le personnel qui relève lui-même son compteur en fin de période et signe le total sur une feuille attachée au compteur. Chacun doit avoir la possibilité de savoir chaque jour, tout au long de la semaine ou de la période admise pour les reports, où il en est et de quelle durée il est redevable au créditeur.

Pour cela les feuilles de présence doivent être facilement accessibles et lisibles et les compteurs, quand ils existent, placés dans un couloir proche du bureau ou dans le bureau lui-même; en tout cas dans un lieu de passage ou les intéressés... et les collègues puissent voir les résultats. Dans de nombreuses entreprises on a préféré que ces compteurs ne soient pas sous le contrôle du chef et que le contrôle hiérarchique soit remplacé par un contrôle collectif.

Les contrôles de la direction se font généralement au moyen de sondages!

Il existe toujours des fraudes; mais elles sont vite décelées et il est rare qu'elles excèdent 1 à $2 \%$ du personnel. La gestion autonome de ces temps de présence a d'ailleurs fait apparaître dans certaines entreprises (Helmer) qui ont procédé à des contrôles systématiques, qu'après plusieurs mois de ce régime, $55 \%$ de l'effectif avait un crédit d'heures $(17 \%$ avait entre 3 et $5 \mathrm{~h}$ de crédit et $33 \%$ avaient plus de 5 heures) et que $10.8 \%$ seulement étaient débiteurs $(56 \%$ de 1 heure : $9 \%$ entre 3 et 5 heures). 
Quant au personnel qui déborde l'horaire flexible et empiète sur l'horaire fixe il ne s'élevait pas à plus de 2,4\% des effectifs globaux, services commerciaux compris!

La firme Messerschmidt s'est même retrouvée en fin d'année avec un total de 37,000 heures qui n'avaient pas été récupérées. Il est très important de démythifier la notion de pointage et de faire comprendre au personnel qu'il ne s'agit que d'un constat de temps et d'une autogestion de celui-ci pour laquelle on lui fait confiance! Il existe d'ailleurs un système de compteurs individuels (Hengstler) qui ne comporte pas d'enregistrement de l'heure d'arrivée ou de départ, mais seulement un décompte du temps fait et dû.

Cependant dans tous les secteurs où il n'existait pas au préalable de contrôle strict des horaires, on peut se heurter à des difficultés d'application! Nous pensons néanmoins que ce système présente assez d'avantages (diminution du sentiment de culpabilité, de l'anxiété, de la nécessité de se presser etc....) pour être accepté après un certain temps de rodage. Le personnel est très sensible à la suppression des sujétions imposées par un horaire fixe. C'est dans cet esprit et enfin aussi d'éviter toute discrimination qu'il est utile d'appliquer le même régime aux cadres, en dépit des réticences et du refus de ceux-ci. Dans la plupart des entreprises les cadres jusqu'au niveau des cadres supérieurs se sont pliés à cette exigence même quand ils ne pointaient pas auparavant. Cependant, en France, à la Caisse Centrale de Prévoyance Mutuelle Agricole par exemple aucun cadre ne gère lui-même son temps et n'est soumis à ce contrôle. En revanche, chez Peugeot, même le Directeur Général enregistre son temps. Quand on a du mal à obtenir des cadres qu'ils acceptent un compteur individuel, on peut leur donner une simple feuille de présence individuelle sur laquelle ils marquent leurs heures d'arrivée, de départ et leurs déplacements. Mais l'expérience montre qu'ils en ressentent vite une grande lassitude et réclament d'euxmêmes un compteur.

Il est également important pour les cadres et pour les directions que les cadres apprennent à gérer leur temps et prennent conscience du temps passé dans leur bureau et hors de celui-ci. Très souvent les directions exigent de leurs cadres un long temps de présence; mais en même temps les proches collaborateurs de ceux-ci et ceux qui sont sous leurs ordres prennent cette durée excessive pour une provocation et la supportent mal. L'enregistrement des temps est un excellent moyen de prise de conscience. 


\section{Les moyens de contrôle}

Parmi les modalités de contrôle on peut citer :

\section{La feuille de présence, affichée ou non}

C'est le moyen le plus simple et le moins onéreux. Mais ces feuilles de présence sont mal vues et souvent considérées comme une brimade. Pour des raisons psychologiques il serait mieux, semble-t-il, d'utiliser un moyen qui ne soit pas marqué d'une image aussi défavorable (et particulièrement le compteur individuel).

De plus, quand les reports sont autorisés et le personnel nombreux, elles deviennent difficiles à exploiter.

Enfin, il se manifeste très vite un sentiment de lassitude. Au bout d'un certain temps on ne signe plus.

On peut aussi utiliser l'annotation individuelle sur des bulletins délivrés chaque semaine. Mais il s'agit d'une méthode incontrolable quant à ce qui est noté sur les fiches, et chacun perd du temps à établir son calcul journalier. Cette méthode éveille en outre la méfiance car personne ne sait plus ce que l'autre a enregistré et il en résulte vite, comme souvent aussi avec les feuilles de présence, une détérioration du climat social.

\section{L'HORLOGE POINTEUSE}

Elle appelle les mêmes remarques. Sur le plan psychologique elle est aussi très mal acceptée par le personnel qui supporte mal la contrainte de la file, et la considère comme dégradante.

Cependant certains systèmes, au lieu d'indiquer l'heure d'arrivée et de départ enregistre le temps de travail effectif de la journée. Chacun possède sa carte individuelle de pointage. Les retards apparaissent en rouge avec le temps effectif de retard.

Dans une entreprise où le personnel n'avait jamais pointé et qui comptait beaucoup d'éléments «turbulents», au bout de six mois le système était accepté sans réticences.

L'avantage de cette horloge est de pouvoir fournir en plus du contrôle du temps une masse de renseignements (absences, temps passé hors de l'entreprise ; etc). 
L'UTILISATION DE COMPTEURS SPÉCIAUX INDIVIDUELS

Ils comptabilisent la situation de chaque salarié. Ces compteurs enregistrent le temps de présence par l'introduction d'une carte personnelle ou badge analogue à une carte de crédit et qui reste en place pendant tout le temps de travail. Le total est reporté en fin de semaine ou de mois et le compteur remis à zéro.

Les heures d'arrivée et de départ n'étant pas enregistrées donc pas contrôlables par le chef, le compteur individuel donne au salarié le sentiment de liberté. Seules sont comptabilisées en centièmes d'heure les heures de présence.

Ce système qui est de lecture facile permet un contrôle collectif des heures du voisin et de celles du chef. C'est un élément qui met fin à la méfiance habituelle et aux rumeurs sur le travail des autres.

Le compteur est relevé une fois par semaine ou par mois et les feuilles de décompte arrivent déjà toutes exploitées au service de comptabilité et du personnel, le travail administratif se trouve ainsi simplifié !

Chaque compteur est équipé d'une lampe témoin qui s'allume quand on introduit le badge. Ce qui permet au chef de service de détecter d'un simple coup d'œil les absences.

Certains badges sont personnalisés, de telle sorte qu'on ne peut pas mettre n'importe quel badge dans un compteur; ce qui évite aussi les resquilles ou les plaisanteries, ces dernières étant d'ailleurs sévèrement sanctionnées quand d'aventure elles se produisent.

Sur le plan psychologique ce badge est la concrétisation de l'autonomie et de la liberté du personnel.

Enfin il existe des systèmes faisant appel à l'informatique et intégrés à la gestion automatisée des opérations concernant le personnel.

Quand on veut tirer des compteurs individuels une masse de renseignements ou encore alléger le travail administratif (dans le cas où il s'agit de gérer des effectifs importants) on peut le relier à un ordinateur.

Cependant il faut noter qu'en France tout au moins ce relais par ordinateur est très mal accepté par le personnel. D'une part le personnel redoute la manipulation ou les erreurs d'un appareil dont il ne peut vérifier le fonctionnement, d'autre part il accepte mal de recevoir un état de contrôle avec 15 jours ou 3 semaines de retard. Ce qui l'inté- 
resse c'est de savoir toujours où il en est; et c'est aussi la liberté apparente qu'il garde dans son travail et qui lui fait refuser de voir cette liberté mise sur carte et contrôlée par une machine.

Il s'agit là d'une difficulté qui a été ressentie avec une quasi unanimité. En réalité, il faut avant tout savoir ce que l'on attend de ce constat de temps.

Si l'on veut seulement s'assurer du nombre d'heures de présence, des moyens simples et peu onéreux suffisent... sous les réserves psychologiques faites précédemment.

Si l'on veut obtenir des renseignements plus élaborés, tenir des statistiques, faire de la gestion de personnel automatisée, il est nécessaire d'avoir recours à un ordinateur. Les «sous-produits » élaborés par l'ordinateur sont nombreux et variés et ils dépassent les problèmes d'horaires flexibles.

\section{Sanctions}

En cas de retard, c'est-à-dire d'empiettement sur la partie fixe, des sanctions peuvent être prises. Elles ne le sont en général que si le retard est répété.

Dans ce cas la sanction pratiquée est rarement financière. Elle consiste généralement à remettre pour un temps déterminé le retardataire à l'ancien horaire fixe.

Enfin il faut signaler que certains salariés, rares il est vrai, se sont révélés incapables d'autogérer leur temps et ont dû être remis aux horaires fixes.

\section{Les absences}

L'application de l'horaire flexible a pour conséquence une très nette diminution des absences pour convenance personnelle, notamment des absences de courte durée. Avec les horaires fixes les salariés qui se trouvaient en retard préféraient souvent ne pas venir et perdre $1 / 2$ journée de travail que de s'exposer à des remarques ou à des sanctions. Dans certaines entreprises on a noté une diminution de $80 \%$ des absences (Helmer). En Suisse on estime qu'elle se situe entre 40 et $55 \%$ ! Ailleurs elles ont complètement disparu (Produits Roche).

Chez Peugeot on a constaté qu'en une année, la réduction de l'absentéisme couvrait les frais d'investissement des appareils. 
Un des avantages des horaires flexibles est d'établir un rééquilibrage du temps. Le personnel est souvent plus disposé à prendre à sa charge certaines absences qu'il laissait à l'entreprise (banque, médecin, etc.). C'est le cas notamment des petits retards dus aux incidents de transport. Très souvent quand le retard est de peu d'importance, le personnel préfère le récupérer que de perdre encore du temps à réclamer un billet de retard. Souvent aussi il préfère s'épargner l'humiliation de demander une « faveur ».

Ce point a d'ailleurs éveillé les inquiétudes des syndicats qui craignaient que les facilités données ne se soldassent par la suppression de certains avantages tels que certaines absences pour motifs personnels ou familiaux tolérées ou reconnues par convention et par conséquent payées.

Sur ce point les attitudes des entreprises sont très diverses. En France la tendance a été de maintenir le régime existant des absences payées pour motifs personnels ou familiaux tel qu'il était prévu dans les accords d'entreprises ou les usages. Aussi certaines entreprises n'imposent pas la récupération quand le salarié s'est trouvé malade sur les lieux du travail et obligé de quitter celui-ci, ou quand sur le lieu de son travail il apprend le décès ou la maladie d'un proche exigeant sa présence etc.

D'autres acceptent de donner des bons de sortie pour cas exceptionnels. En ce qui concerne les retards occasionnés par les grèves, certaines entreprises (Helmer), après accord avec le délégué du personnel prennent à leur charge les heures perturbées de ce fait mais n'acceptent pas d'autres dérogations. Peugeot en revanche ne les accepte pas.

En fait il s'agit d'autant de cas d'espèce et il n'y a pas de cloctrine générale.

Il faut prévoir ces modalités dans le réglement intérieur et si possible avec le personnel, en partant de ce préalable que l'institution d'horaires flexibles ne doit pas être une source d'anarchie.

En dehors de ces absences pour convenance personnelle, il y a toutes celles qui sont dues à des travaux ou réunions exécutés à l'extérieur! Celles-ci se présentent fréquemment pour les cadres.

Plusieurs solutions peuvent être adoptées ... Dans le cas de compteur individuel, si l'intéressé revient à son bureau après son passage à l'extérieur il laisse son badge engagé. Sinon il l'enlève et inscrit à la 
main ses heures et motifs d'absence. Il en est de même avec les fiches d'horloge pointeuse ou les fiches manuelles. Les absences, déplacements pour service et temps divers, non enregistrés par le compteur sont notés directement au jour le jour sur le relevé hebdomadaire. Celui-ci permet de s'assurer, en fin de période, que l'horaire dû ( 40 heures) a bien été respecté.

Toutes les entreprises ont des problèmes en ce qui concerne ces travaux extérieurs (services commerciaux, services après vente, etc.). Elles ne s'illusionnent pas sur l'efficacité d'un contrôle; mais pensent qu'il faut faire confiance à leur personnel et ne pas lier le temps de présence au temps payé.

Quand les absences chevauchent sur deux périodes, (voyages, maladie etc.) on demande dans la mesure où elles sont prévisibles d'informer avant de partir le service qui gère le temps.

\section{Accidents du trajet}

Les entreprises qui ont appliqué les horaires flexibles ont constaté une diminution, voire une suppression des accidents du trajet. Cependant en ce qui concerne ces accidents, des difficultés sérieuses peuvent surgir avec la législation en vigueur sur les accidents du travail.

La jurisprudence précise qu'il doit être en rapport direct et immédiat avec le travail qui va avoir lieu ou qui vient de s'accomplir.

Quand les horaires sont imposés il est facile de déterminer si l'accident est bien survenu dans le temps normal du trajet. Dans le cas d'horaires flexibles, l'accidenté devra généralement démontrer que son trajet était bien en rapport direct et immédiat avec son travail.

Si l'accident a lieu au retour du travail, l'assuré ayant pointé à son départ on saura avec certitude à quelle heure a commencé son trajet.

Si l'accident a lieu à l'aller plusieurs cas peuvent se produire :

- ou bien l'heure d'arrivée du lendemain avait été portée à la connaissance de l'employeur, et pourvu que l'accident survienne dans un temps conforme à cet horaire, il n'y a pas de problèmes. C'est la raison pour laquelle certaines entreprises demandent ainsi à leur personnel d'indiquer à l'avance, d'une semaine sur l'autre l'heure présumée d'arrivée ou de départ.

- ou bien l'heure de prise du travail est laissée au libre choix du salarié et dans ce cas il peut y avoir contestation. 
a) s'il a l'habitude d'arriver tous les jours à la même heure, (c'est le cas de $75 \%$ des salariés) grâce à ses fiches de contrôle il pourra prouver le caractère normal de son trajet et de l'heure de l'accident.

b) s'il arrive à des heures variables il devra apporter la preuve qu'il se rendait effectivement et directement à son travail. Dans ce cas il devra recourir au témoignage des voisins, des collègues, à l'attestation de son chef de service. L'employeur devra informer la Sécurité Sociale qu'il pratique les horaires libres et préciser dans la déclaration d'accident que l'employé était bien dans les limites tolérées.

\section{LES RÉACTIONS DES CADRES}

Si l'horaire flexible est très bien accepté par le personnel, il soulève en revanche beaucoup de réticences chez les cadres.

Les cadres qui n'étaient pas astreints au pointage acceptent mal un contrôle de leur temps. Ils le jugent dégradant et s'estiment rabaissés au niveau des cols bleus. Ils redoutent que l'on veuille contrôler leur temps.

Mais l'entreprise n'a aucun intérêt à faire montre d'un esprit policier. Le plus souvent quand elle a rassuré les cadres sur ce point, les difficultés disparaissent.

En outre, ce contrôle est très utile, nous l'avons dit, car il est bon que cadres et directions sachent comment les premiers utilisent leur temps. Dans la majorité des cas ni les uns ni les autres n'en ont une notion claire.

Cet auto-contrôle permet aussi aux cadres de mieux organiser leur temps et de le mieux répartir notamment entre l'extérieur et l'intérieur, et souvent de se discipliner, notamment dans leurs rapports avec leurs subordonnés. Il révèle ainsi aux directions les qualités de leurs cadres. Celles-ci ne doivent pas se juger au nombre d'heures qu'ils accomplissent et qui nous l'avons dit est souvent mal vu du personnel, mais à leur efficacité et au contraire à la façon dont ils arrivent à accomplir le travail dans le temp imparti. L'entreprise Helmer considère comme une grave erreur de penser que les cadres doivent avoir un temps de présence plus long que les autres salariés. Ayant davantage de responsabilités ils devraient au contraire avoir besoin d'un plus long temps de disponibilité intellectuelle.

Mais cela pose tout le problème des directions et de leur mentalité. 
Nombreux aussi sont les cadres qui ne supportent pas de n'avoir pas tout leur personnel présent quand ils sont là. C'est dans ce domaine que l'auto-discipline est la plus nécessaire. Il est rare qu'un cadre ait besoin toute la journée, de tout son personnel et il peut, le plus souvent sans difficultés, reporter sur les plages fixes, ses exigences à l'égard de celui-ci. Les établissements Helmer ont fait une enquête par sondage et constaté que $15 \%$ seulement en moyenne du temps journalier et $25 \%$ au maximum était nécessaire aux communications.

Enfin certains cadres, tatillons et tyraniques, trouvent dans le contrôle des horaires stricts, une occasion d'exercer à peu de frais leur autorité et n'apprécient pas qu'on leur enlève cette parcelle de pouvoir.

Quoi qu'il en soit, si les horaires libres se soldent jusqu'ici par une quasi totalité de succès, ils ont connu au moins un échec qui a été provoqué par le refus des cadres de participer à l'expérience.

En tous cas lors de la phase préparatoire à l'introduction des horaires libres, il faut faire un effort particulier d'information et de persuasion au niveau des cadres.

\section{LES RÉACTIONS DES SYNDICATS}

$\mathrm{Au}$ début de l'expérience les syndicats se sont montrés réticents. Ces expériences ont toutes été tentées à l'initiative patronale et cela suffisait à éveiller leur méfiance.

Leurs arguments varient selon les centrales et les entreprises. On peut, en gros les résumer ainsi :

L'objectif majeur demeure la réduction de la durée du travail et l'introduction de l'horaire libre ne doit pas être un moyen détourné de maintenir la durée actuelle.

Ils craignaient aussi de voir remises en cause les absences familiales ou personnelles ainsi que les heures supplémentaires et que le patronat n'en profite pour récupérer à son seul profit un certain nombre d'avantages concédés aux travailleurs. De même craignaient-ils, tout comme l'Inspection du Travail, qu'à la faveur des horaires libres, ne soit mise en péril la protection que la législation actuelle assure aux travailleurs.

Ces craintes semblent jusqu'ici avoir été vaincues. 
En revanche ils estiment que ces horaires laissent moins d'occasion de rencontre et diminuent la fréquence des contacts humains; ils estiment aussi que la plus grande responsabilité laissée aux travailleurs constitue une subtile contrainte morale.

Néanmoins ces réticences n'ont jamais été jusqu'au refus et on ne connaît pas de mouvement concerté de protestation contre ce système.

\section{COÛTS}

Il existe sur le marché un nombre relativement important de systèmes de contrôle et leur prix varie avec l'étendue des services qu'ils peuvent rendre. Les horloges fonctionnent individuellement ou en batterie. La dépense par personne s'établit entre 25 et 40 dollars selon les systèmes employés. Avec ordinateur la dépense peut atteindre 120 à 150 dollars pour le système le plus évolué.

\section{COMMENT INTRODUIRE UN SYSTÈME D'HORAIRES VARIABLES ?}

1) La première des règles qui se dégage des expériences faite tant en France qu'à l'étranger c'est qu'il faut agir avec prudence, par petits secteurs et non sous la forme d'une injection massive. Il est préférable de tenter d'abord une expérience dans des secteurs déterminés et sur de petits groupes comportant de 15 à 30 personnes et de la tester, au bout de 2, 3 ou 4 mois. En effet, on ne sait pas d'avance comment les changements seront perçus par le personnel et on peut, en testant noter le degré de satisfaction de celui-ci, la marche du service, le pourcentage des absences, retards, etc.

Aussi la maison Sulzer à Winter-Thour a commencé un essai dans 3 départements occupant 700 salariés. Les expériences s'étant avérées concluantes, l'essai a été étendu à environ 2,000 personnes. Enfin, il fut décidé de l'introduire peu à peu dans toutes les entreprises et filiales du groupe, partout où cela pouvait se faire sans nuire au déroulement des opérations, et aujourd'hui 8,000 personnes bénéficient de l'échelle horaire mobile.

Cette innovation nécessite des analyses de structures et d'organisation très approfondies, une appréciation des préférences du personnel, l'examen des questions juridiques, psychologiques, etc.

Il est indispensable de faire participer le personnel et ses représentants à l'étude des problèmes qui se posent et à la programmation. 
2) Il faut aussi faire montre de patience. La plupart des entreprises ont signalé des difficultés de mise en route avant que le système fonctionne convenablement (difficultés de communications, départ de la plus grande partie du personnel dès l'heure limite quand il fait beau ou le vendredi soir, arrivée tardive le lundi matin etc.) résolues.

Mais au bout d'un certain temps de rodage, ces difficultés se sont

3) Il faut enfin accepter des tatonnements et laisser au personnel le temps de s'habituer à se servir des appareils d'enregistrement et d'apprendre à autogérer son temps. On ne passe pas spontanément d'un régime autoritaire et militaire à un régime autonome.

Il est recommandé aussi de commencer par les secteurs ou services réputés les plus difficiles. Si l'expérience réussit dans ceux-ci elle aura toutes les chances de pouvoir être étendue aux autres. Aussi voit-on de nombreuses entreprises commencer leur expérience par le service de la comptabilité.

4) Un règlement intérieur doit être établi pour régler les grandes lignes de l'expérience et éviter les malendus (notamment en ce qui concerne les absences, les heures supplémentaires, les plages, les pauses du déjeuner etc., les retards, les déplacements, les modalités de récupération etc.).

Mais ce règlement doit se garder de vouloir tout prévoir et réglementer. Un certain nombre de difficultés ne peuvent être résolues que lorsqu'elles se présentent et selon les circonstances du moment. Un règlement rigide impose des contraintes qui nuisent souvent à l'efficacité des solutions.

5) De l'aveu de tous ceux qui ont tenté ces expériences, la règle d'or est le pragmatisme. Il faut éviter le perfectionnisme, le souci du détail tatillon et régler les problèmes quand ils surgissent.

6) Il faut assurer une distinction précise entre les heures excédentaires et les heures supplémentaires, par exemple en obligeant l'employeur ou le chef de service à indiquer par une note de service les heures supplémentaires qu'il demande au salarié en plus de son horaire et qui devraient être payées au tarif majoré.

7) Il est nécessaire de limiter de façon précise les débits d'heures normales qui peuvent être reportées d'un mois sur l'autre. On les limite généralement à 10 heures; quelquefois à 15 heures. 
8) Il faut aussi limiter les heures excédentaires pour convenance personnelle (non payées au tarif majoré) à 5 heures par semaine en plus de l'horaire maximal de 50 heures par semaine afin de ne pas enfreindre la législation du travail.

9) Enfin il faut respecter la durée maximale du travail journalier de chaque salarié, fixée actuellement à 10 heures (sauf pour les jeunes de moins de 18 ans).

10) Tous ces points doivent figurer dans le règlement intérieur qui doit être simple et aussi bref que possible, 1 à 4 pages recto verso. Il ne dois pas chercher à résoudre les cas qui ne se produiront qu'une fois. Mais pouvoir au contraire s'appliquer à tous.

11) La partie la plus importante de la mise en place des horaires libres réside dans l'information $=$ information des employeurs, des directions et des cadres d'une part qu'il faut non seulement informer mais convaincre, information du personnel d'autre part.

\section{L'information}

Cette information doit être claire, large, précise, et apportée au moment opportun.

- Il est préférable de réunir de petits groupes afin qu'ils puissent poser des questions; mais il est indispensable qu'il y ait au moins un cadre présent dans chaque unité.

- Dans une première réunion (qui peut réunir un groupe plus vaste) on donnera seulement les grandes lignes de l'opération, les principes, l'esprit dans lequel elle est faite.

On s'efforcera aussi d'expliciter la terminologie qui est loin d'être fixée. Il faut que chacun sache avec précision et netteté de quoi on parle.

- Au départ, il sera utile de bien marquer les limites de ce que l'on va étudier en précisant qu'il ne s'agit que d'une étude et pas d'une opération définitive.

- Il faut donner une information large, globale, et non une information fragmentaire qui déclencherait des rumeurs et risquerait d'être mal interprétée et d'éveiller la méfiance. Mais pour cette raison, il ne faut la donner que lorsque le problème est arrivé à maturité et que la solution choisie a toutes les chances d'être adoptée.

- Chaque entreprise ou chaque service doit ensuite mener l'information de son personnel selon la nature du service ou de la maison, ses 
traditions, son état d'esprit. Il n'existe pas deux services ou deux entreprises identiques et il n'y a pas de recettes toutes faites.

- Enfin, il faut répéter plusieurs fois l'information, car l'expérience est rarement comprise du $1 \mathrm{er}$ coup et il faut avant de la tenter que le personnel comprenne bien le mécanisme de l'autogestion, des reports, du décompte du temps (qui est composé en centièmes d'heure, etc.). À la Caisse Centrale de Prévoyance Mutuelle Agricole par exemple, le directeur lui-même a été dans tous les services pour expliquer le fonctionnement des horaires libres.

- On peut faire cette information en engageant une personne qui fera toute l'étude et la répercutera dans les services; ou encore utiliser pour ce faire les éléments moteurs de chaque service qui feront l'information dans leur département. Cette dernière formule donne de meilleurs résultats car le personnel a l'impression que c'est lui qui crée le système.

- On peut également remettre avec le bulletin de paye une note faisant le point de la question et un questionnaire. Mais cette note ne dispense pas de l'information orale nécessaire pour que chacun puisse poser des questions et pour que les chefs puissent s'assurer que l'expérience a été bien comprise.

- Enfin il est nécessaire d'informer des nouveaux projets les représentants du personnel et le comité d'établissement quand il existe et si possible d'avoir au préalable leur accord.

\section{Les étapes de la réalisation}

En résumé et sur le plan pratique quand une entreprise veut établir les horaires libres elle doit procéder en plusieurs étapes :

1) Procéder à l'étude préalable et approfondie de la question, et faire des sondages auprès des principaux intéressés pour voir comment ils accueilleront cette suggestion.

2) Informer le personnel au cours d'une première réunion et le préparer psychologiquement.

3) En conformité avec la législation, recueillir l'avis du comité d'entreprise nécessaire pour toute modification d'horaire. (Mais l'employeur n'est pas tenu de suivre cet avis). Par ailleurs il n'est pas recommandé de faire faire l'information par le comité d'entreprise.

4) Prendre contact avec les organisations syndicales pour connaître leurs réactions. 
5) Faire de nouvelles séances d'information par petits groupes afin que directions, cadres et salariés, adoptent une attitude positive à l'égard de l'expérience... à tout le moins une attitude, d'attente et de neutralité.

6) Établir un réglement intérieur et le soumettre au personnel.

7) Une fois la décision prise, prendre contact avec l'inspection du travail et lui indiquer les modalités des nouveaux horaires.

8) Tenter l'expérience sur un petit secteur déterminé et faire un test de présence, d'efficacité, de satisfaction, au bout de 3 ou 4 mois.

9) Si l'expérience a réussi, l'étendre progressivement et non de façon massive à d'autres services.

\section{LIMITES}

L'horaire mobile n'est pas praticable dans toutes les entreprises ou tous les postes de travail. Mais on aurait tort de croire qu'il ne s'adapte qu'au secteur administratif, n'ayant pas de rapport avec le public. Le personnel qui ne peut pas en bénéficier est beaucoup moins nombreux qu'on ne le croit habituellement.

C'est ainsi que dans une fonderie de précision où les hauts fourneaux travaillent en équipes et par groupes de 5, on a laissé aux groupes eux-mêmes le soin de prendre la décision concernant les horaires de travail. Mais les groupes ont si bien planifié leurs horaires qu'il. n'y a jamais eu de perturbation dans le processus de production.

Dans des entreprises de travail à la chaîne on a introduit des stockstampons, calculés avec précision afin de pouvoir parer à l'absence de travailleurs individuels pendant la phase mobile des horaires.

Mais il est évident que ces solutions ne peuvent être trouvées qu'après une analyse approfondie da le situation, des possibilités d'organisation, du degré d'interdépendance des différents postes de travail, etc.

Les horaires libres sont une facilité accordée par les entreprises à leur personnel et sont conformes aux nouvelles exigences de celui-ci qui demande plus de responsabilité et d'autonomie.

Mais ils ne sont pas une panacée et ne résolvent pas tous les problèmes de personnel. Ils ne doivent pas non plus être un alibi qui permettra de masquer la réalité d'autres revendications, notamment de celles tendant à la réduction de la durée du travail. 


\section{Flexible Hours}

For the last five years rigid working schedules have increasingly been under attack and many new experiences have been tried in many European countries, particularly in Germany and Switzerland. The essence of these experiences has been to adapt working schedules to each worker through a flexible hour system. The basic principle of such a system is the free and individual choice of entry and exit time within certain limits.

The flexible working hours system generally distinguishes three separate components to a working day : a core time when all employees must be at their job and two flexible time periods, at the beginning and at the end of the working day.

Such a system has advantages for both the employer and the employee.

As for the employer, such a system facilitates time computing, encourages a decrease of short term absenteism and turnover, a reduction of internal traffic, a decrease of accidents on the way to work, a reduction of overtime, greater hiring advantages, and a longer service to the consumer. Moreover management workers may work in a more peaceful atmosphere, the social climate is ameliorated and internal conflicts are reduced.

As for the employees, a reduction of transportation time has been noted, family and personal needs are given a better chance to be satisfied, and a change of attitude towards work was underlined.

There is no best way to design and install such a system. There are many variations to it and adaptation is surely favorized under such a system. Experience, however, teaches some ground rules. First such a system much be progressively introduced. Experimentation is greatly encouraged. Second, it takes time for the system to give good results. Third, trial and error is often the best method. Fourth, rules must be established to avoid misunderstandings. Firfth, pragmatism is the golden rule. Sixth, a clear distinction must be made between exceeding hours and overtime. Seventh, a lot of information is necessary before and during the introduction of the system.

Flexible working hours practically require a form of control. This control, however, should itself be as flexible and as discreet as possible. Self control is to be aimed at. Such a control can be made by a time card, posted or not, by a punch clock, by special individual meter or by computer.

To the same extent that flexible working hour system is well accepted by employees, to the same extent does it find obstruction at the middle management level. That the system is applied to middle management is perceived as a move to put them on the same level as blue collar workers. But the basic reluctance of white collars comes from the revised notion of authority required by a flexible hour system. If one group needs particular attention when flexible hours are introduced, it is middle management.

Eventhough early reluctance of unions has been greatly dissipated, they still feel that such a system decreases the possibility of human contacts and that the greater responsibility left to the workers is subtle moral constraint.

Up to now, flexible hours have been very successful. This is not to say that they are a panacea nor that they will settle all personnel problems. 